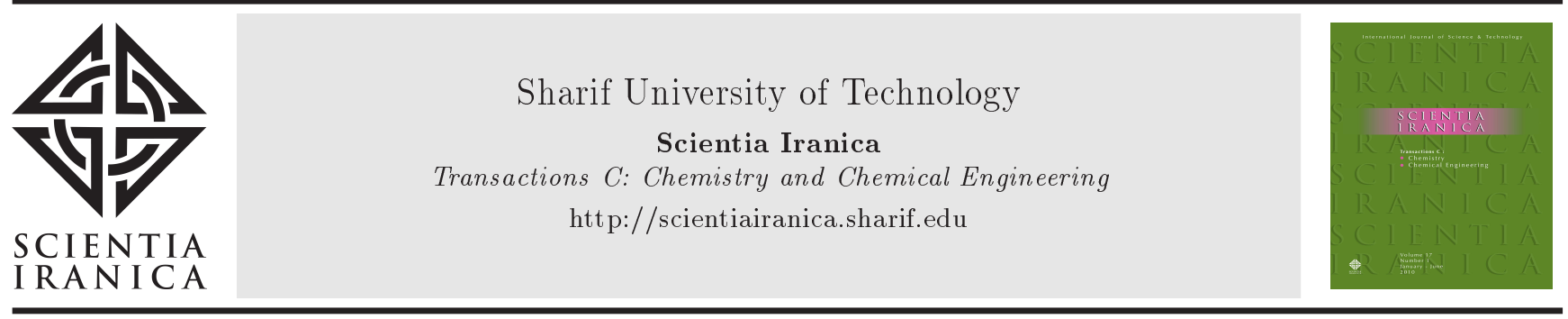

\title{
The effects of bubble detachment shape on rising bubble hydrodynamics
}

\author{
P. Fayzi $i^{a *}$ D. Bastani ${ }^{a}$, M. Lotfi ${ }^{b}$, and M.G. Khararoodi ${ }^{a}$ \\ a. Department of Chemical \& Petroleum Engineering, Sharif University of Technology, Tehran, P.O. Box 11155-9567, Iran. \\ b. Department of Chemical Engineering, Science and Research Branch, Islamic Azad University, Tehran, Iran. \\ Received 20 September 2018; received in revised form 12 November 2018; accepted 8 December 2018
}

\section{KEYWORDS}

Detachment shape;

Aspect ratio;

Local velocity;

Rising bubbles;

Surfactant.

\begin{abstract}
Local velocities and aspect ratios of rising bubbles were measured to investigate the effects of bubble detachment shape on rising bubble hydrodynamics. Two types of capillary were employed to generate bubbles of identical volume: one glassy nozzle aligned vertically and the other stainless steel needle aligned horizontally. Horizontally injected bubbles have a spherical initial shape, and their values of aspect ratio slightly fluctuate around unity. However, vertically injected bubbles have a surface-stretched initial shape, and their values of aspect ratios decrease sharply from 1.1 to 0.65 . There is a notable correspondence between the variation of local velocities and aspect ratios that reflects the relevance of the detachment shape of the bubbles to their surface energy. Correlations of Taylor \& Acrivos and Vakhrushev \& Efremov for aspect ratio were examined by experimental data.

(C) 2019 Sharif University of Technology. All rights reserved.
\end{abstract}

\section{Introduction}

Dynamic interfacial phenomena in the presence of surface active materials have a fundamental role in design, construction, and control of many industrial processes [1-5]. Dynamics of gas-liquid interfaces is one of the most interesting topics in this field. Furthermore, it has a wide scope in terms of applications such as boiling heat transfer, wastewater treatment, and drug delivery [6-8]. There are many chemical processes that involve rising of bubbles through a liquid medium such as distillation, floatation, and evaporation $[9,10]$. For decades, experimental and computational researches were conducted to study the hydrodynamics of the ascending motion of gas bubbles through a liquid phase [11-16]. These studies demonstrate that the motion of a bubble is strongly affected by its size and

*. Corresponding author. Tel.: +98 2166165460

E-mail address: Pouyanfeyzi@gmail.com (P. Fayzi) shape, the physical properties of both phases, and the presence of surface-active substances $[12,17,18]$.

Bubble local velocities and shape deformations have been considered as sensitive parameters in previous studies [19-21]. The presence of surfactants even in traces leads to the significant decrease of rising bubble velocity $[1,20,22,23]$. Dynamic Adsorption Layer (DAL) theory is well recognized to justify this phenomenon. According to DAL, displacement of surfactant molecules at the interface leads to nonuniform surface coverage, and surface tension gradient is generated $[9,24]$. As a result, the mobility of the bubble surface reduces, and friction drag increases due to the creation of Marangoni stresses [25]. Consequently, surface behavior of bubble approaches that of a rigid sphere, and velocity decreases.

The shape of liquid droplets and gas bubbles is affected by the interfacial force balance [26]. Some efforts have been made to investigate the influence of bubble shape deformations on the rising bubble hydrodynamics, drag force, and characteristic trajectories $[21,27,28]$. Variations of surface energy deform 
the bubble shape. Surface energy refers to the product of surface tension and interfacial area, i.e., $E_{s}=S \sigma$, leading to the enlargement of the bubble size, the interfacial area, and consequently surface energy. This makes the interfacial region more unstable; hence, the bubble shape deforms from the highly stable spherical one to oblate, prolate, and so on. Surface active substances could also influence the surface energy by reducing surface tension. It has been found that the rising velocity of 1-2 mm ellipsoidal bubbles in purified water is significantly larger than that of spherical ones with the same volume [29]. However, the effect of surfactants on the bubble detachment shape has been disregarded in previous studies.

Several dimensional analyses have been established to clarify the contribution of forces governing the rising bubble hydrodynamics [30]. These studies [26,31] confirmed that the bubble behavior was governed by the resultant of buoyancy, inertia, surface tension, and viscous forces. The bubble aspect ratio is usually correlated in terms of Reynolds (Re, Eq. (1)), Eötvös (Eo, Eq. (2)), Morton (Mo, Eq. (3)), and Weber (We, Eq. (4)) numbers.

$$
\begin{aligned}
& \mathrm{Re}=\frac{\rho_{l} V d}{\mu_{L}}, \\
& \mathrm{Eo}=\frac{g d^{2}\left(\rho_{l}-\rho_{g}\right)}{\sigma}, \\
& \mathrm{Mo}=\frac{\left(\rho_{l}-\rho_{g}\right) g \mu_{l}^{4}}{\sigma^{3} \rho_{l}^{2}}, \\
& \mathrm{We}=\frac{\rho_{l} V^{2} d}{\sigma} .
\end{aligned}
$$

Tadaki number is also introduced to include all of the mentioned forces, yet with weak dependence on viscosity (Eq. (5)) [30]:

$$
\mathrm{Ta}=\mathrm{ReMo}^{0.23} \text {. }
$$

The inertial and surface tension forces should be taken into account in correlating the bubble aspect ratio for surfactant solutions, because the rising bubble velocity and surface tension are significantly altered in the presence of surfactants. Hence, Weber and Tadaki numbers are appropriate choices to correlate bubble aspect ratios. It has been correlated well by Taylor \& Acrivos [36] in terms of Weber number and by Vakhrushev \& Efremov [37] as a function of Tadaki. Thus, these two models are preferred to be assessed in the case of surfactant solutions.

Gas injection method has been considered as a factor that affects the bubble behavior [30]. It has been shown that the bubble growth rate using substrate nozzle is greater than that of needle [32]. However, the relation between the rising bubble hydrodynamics and the detachment shape of bubbles has not been considered. Although it was shown that velocity variations corresponded with shape oscillations [33,34], the influence of surfactants on the initial oscillation has not been addressed yet.

The aim of the current study is to reveal the effect of surfactants on rising bubble hydrodynamics through shape deformation of the detached bubble. One ionic surfactant SDS (sodium dodecyl sulfate) and the other non-ionic $\mathrm{C}_{10} \mathrm{DMPO}$ (decyl dimethyl phosphine oxide) were used in the experiments. Two types of capillary were employed to generate a fixed volume of bubbles with spherical and surface-stretched shapes in the liquid phase. The present work also aims to examine the Taylor \& Acrivos and Vakhrushev \& Efremov correlations for the aspect ratio of a $1.75 \mathrm{~mm}^{3}$ bubble in surfactant solutions.

\section{Experimental method and materials}

The materials used in the experiments include Sodium Dodecyl Sulfate (SDS) as an ionic surfactant and decyl dimethyl phosphine oxide $\left(\mathrm{C}_{10} \mathrm{DMPO}\right)$ as a nonionic surfactant (the purity of both was about $98 \%$ and both were purchased from Sigma Aldrich). Deionized water with a surface tension value of $72.5 \pm 0.3 \mathrm{mN} . \mathrm{m}^{-1}$ was used as in the continuous phase. Surface tension values were measured by using the pendant drop method. Extra experiments were carried out to check the data reproducibility. The experimental temperature was $25^{\circ} \mathrm{C}$ for all measurements.

The rising bubble method has been frequently used for investigating the dynamics of gas-liquid interfaces $[1,3,20,22,25,35]$. Experimental setup used in this study is shown in Figure 1 . The experiments
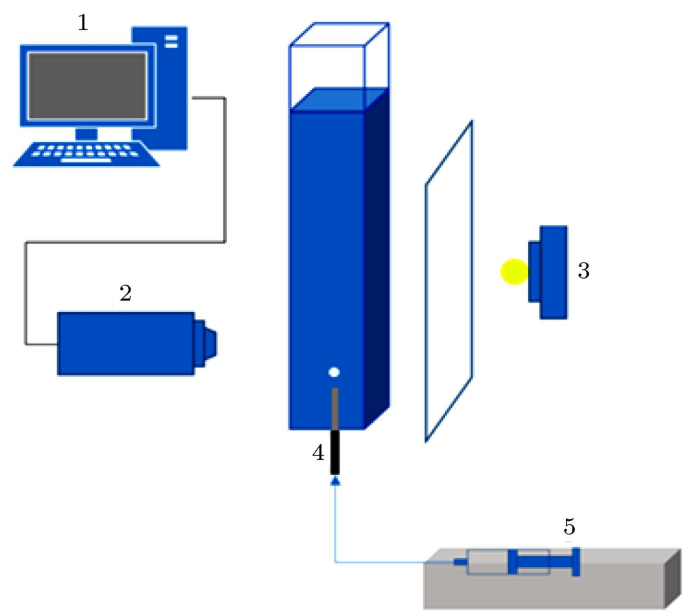

Figure 1. Schematic of the experimental set up: 1: PC; 2: Digital camera; 3: Stroboscope; 4: Capillary nozzle; 5: Syringe pump. 


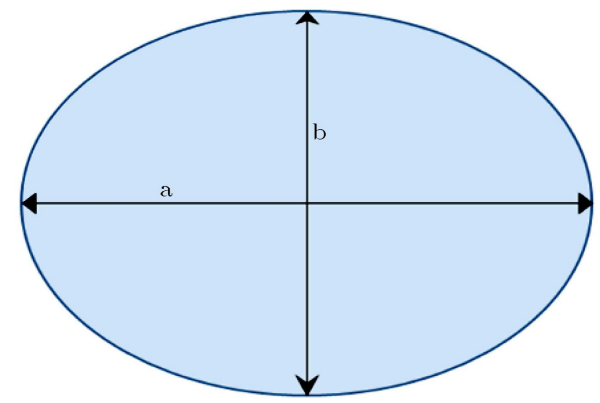

Figure 2. Aspects of ellipsoid form of a rising bubble.

were carried out in a glass square column with a crosssectional area of $40 \mathrm{~mm} \times 40 \mathrm{~mm}$ and a height of $50 \mathrm{~cm}$. Two types of capillaries were used to generate bubbles of the same volume: one glassy nozzle with an inner diameter of $0.075 \mathrm{~mm}$ and aligned vertically; the other stainless steel needle with an inner diameter of $0.1 \mathrm{~mm}$ and aligned horizontally. Capillaries were fitted at the end of the glass square column in a way that the bubbles were produced in the center of cross-section of the column. A syringe pump was used to control the air flow and produce bubbles of $1.75 \mathrm{~mm}^{3}$ volume. A CCD camera was used to record the bubble motion and its deformations. A stroboscope illumination with a frequency of $100 \mathrm{~Hz}$ was employed to specify the bubble position in the corresponding elapsed time. Local velocities were calculated by dividing the displacement of the bubble by the elapsed time.

It is worthy of note that the two-dimensional analysis of bubble shapes has been considered in the current study. The bubble aspect ratio $(E)$ has been calculated according to Figure 2 and Eq. (6) to assess the local shape deformations of the rising bubble:

$$
E=b / a \text {. }
$$

\section{Result and discussion}

Local Velocity Profile (LVP) and aspect ratio $(E)$ of the bubbles rising in pure water and surfactant solutions $\left(10^{-7} \mathrm{M}\right.$ to $10^{-4} \mathrm{M}$ for $\mathrm{C}_{10} \mathrm{DMPO}$ and $5 \times 10^{-5} \mathrm{M}$ to $3 \times 10^{-3} \mathrm{M}$ for $\mathrm{SDS}$ ) were measured. The error of terminal velocity measurements was about $0.4 \mathrm{~cm} . \mathrm{s}^{-1}$. The values of the surface tension measured by the pendant drop technique are listed in Table 1. By increasing the concentration of $\mathrm{C}_{10} \mathrm{DMPO}$, the surface tension decreased, because the highest concentration of $\mathrm{C}_{10}$ DMPO $\left(10^{-4} \mathrm{M}\right)$ was less than its CMC $\left(4.6 \times 10^{-3}\right.$ $\mathrm{M})$. The CMC of SDS was about 7-10 mM; therefore, the surface tension of SDS solutions was also decreased with increasing SDS concentration. Two types of capillary nozzles (one metal needle aligned horizontally and the other one glass nozzle aligned vertically) were used to generate two different initial shapes of detached bubbles (spherical and surface-stretched, respectively) with the same air volume of $1.75 \mathrm{~mm}^{3}$.
Table 1. Surface tension data at various surfactant concentrations.

\begin{tabular}{ccc}
\hline Surfactant & $\begin{array}{c}\text { Concentration } \\
(\mathbf{M})\end{array}$ & $\begin{array}{c}\text { Surface tension } \\
\left(\mathbf{m N . m ^ { - 1 }}\right)\end{array}$ \\
\hline & 0 & $72.5 \pm 0.3$ \\
& $10^{-7}$ & $72.6 \pm 0.3$ \\
$\mathrm{C}_{10}$ DMPO & $5 \times 10^{-7}$ & $72.5 \pm 0.2$ \\
& $10^{-6}$ & $72.3 \pm 0.3$ \\
& $5 \times 10^{-6}$ & $71.4 \pm 0.3$ \\
& $10^{-5}$ & $70.7 \pm 0.2$ \\
& $10^{-4}$ & $61.7 \pm 0.4$ \\
\hline SDS & $5 \times 10^{-5}$ & $72.5 \pm 0.3$ \\
& $10^{-4}$ & $71.2 \pm 0.4$ \\
& $3 \times 10^{-4}$ & $69.7 \pm 0.3$ \\
& $3 \times 10^{-3}$ & $57.2 \pm 0.3$ \\
\hline
\end{tabular}

\subsection{Correlating the bubble aspect ratio}

There are various models for correlating the bubble aspect ratio. The aspect ratio depends on the forces that act on the rising bubble and has been correlated as a function of Re, Eo, Mo, and We. The velocity and surface tension of the rising bubble are considerably changed in the presence of surfactants, and the bubble shape is mainly controlled by inertia and surface tension. Hence, for correlating the bubble aspect ratio in surfactant solutions, the inertial and surface tension forces may be accounted in terms of We and Ta, respectively. Taylor \& Acrivos [36] presented a Weber-based correlation (Eq. (7)), which was examined successfully by Celata et al. [30]:

$$
E=\frac{1}{1+\frac{5}{32} \mathrm{We}} .
$$

Vakhrushev \& Efremov [37] revised the Tadaki and Maeda correlation as follows:

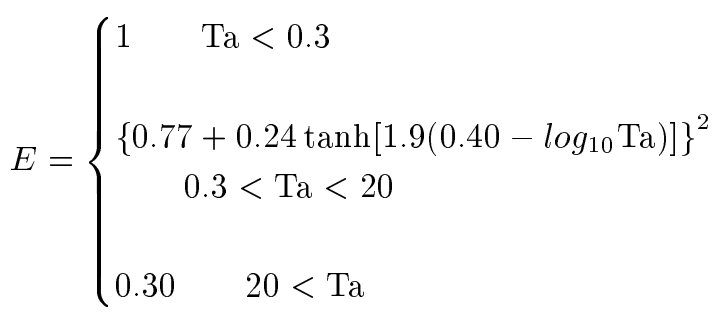

The accuracy of Eq. (8) also was confirmed by Celata et al. [30].

\subsection{The effects of bubble injection method}

The aspect ratios and LVPs of the rising bubbles detached from vertical nozzle and horizontal needles in pure water are shown in Figure 3. According to Figure 3 , the bubble detached from the horizontal needle is approximately spherical $(E \approx 1)$, and its aspect 


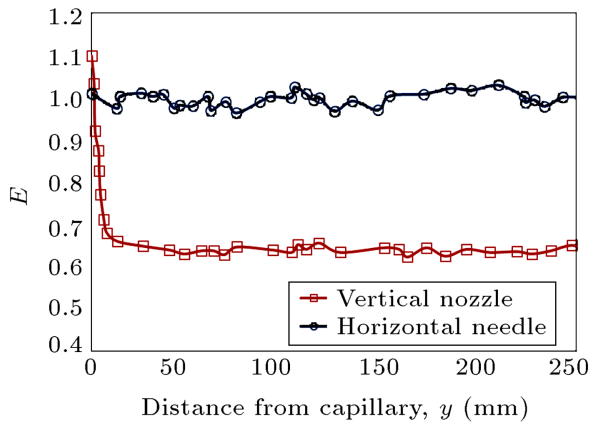

(a)

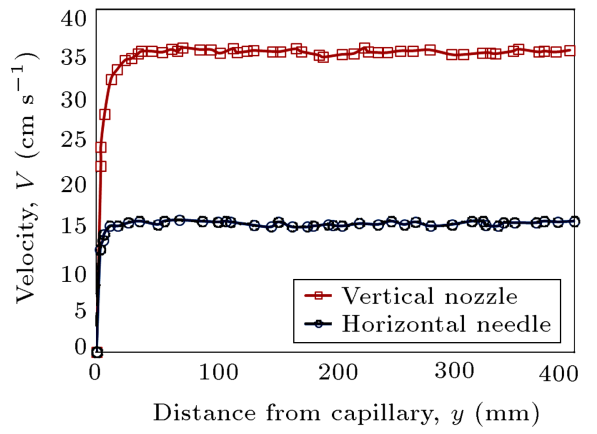

(b)

Figure 3. Hydrodynamic behavior of rising bubbles in pure water: (a) Aspect ratio profile and (b) local velocity profile.
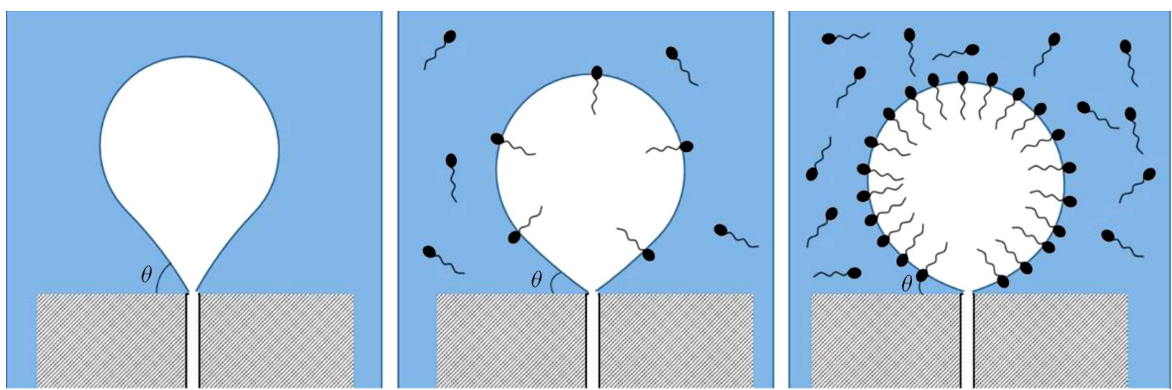

Figure 4. Schematic effect of surfactant molecules on detached bubble shape of a rising bubble.

ratio fluctuates slightly about unity along the rising path. In other words, the bubble rises without any significant deformation of its initial spherical shape. However, the bubble detached from vertical nozzle in pure water has a surface-stretched shape, and its aspect ratio sharply decreases from 1.1 to 0.65 . As illustrated in Figure 3(b), there is an acceleration step in LVPs of vertically and horizontally injected bubbles, followed by constant (terminal) velocity. However, local velocities of vertically injected bubbles have higher values than those of horizontally injected bubbles. The acceleration step of vertically injected bubble occurs along with the sharp decrease of aspect ratio. It is also deduced from Figure 3 that the bubble terminal velocity is strongly dependent on its detachment shape. Thus, it appears that the initial shape of the detached bubble could affect the LVPs and aspect ratios of the rising bubbles. The interfacial area of surface-stretched bubbles is higher than that of spherical bubbles with the same volume. Thus, the surface energy of vertically injected bubbles has higher values than that of horizontally injected bubbles. This disparity between surface energies could be responsible for different LVPs of rising bubbles in two injection methods.

\subsection{Bubble detachment shapes}

Variations of surface energy create different curvatures of the bubble surface during the detachment process. The presence of surfactant molecules leads to decreasing bubble surface energy by reducing the gas-liquid

surface tension. Hence, the interfacial curvature tends toward stable spherical shape rather than unstable surface-stretched shape. This behavior is illustrated in Figure 4. Surface-stretched bubbles have more surface energy than spherical ones due to their higher interfacial area. In other words, a spherical shape is the most stable form for the detachment curvature of rising bubbles with the same volume. As is shown in Figure 5, during bubble detachment from the vertical nozzle, the instability of the bubble surface makes it oscillate.

As illustrated in Figure 6, with the increase of surfactant concentration, the initial shape of vertically injected bubbles approaches a spherical shape. For vertically injected bubbles in pure water, the aspect ratio is sharply decreased to a constant value less than 1. As is seen, horizontally injected bubbles possess a higher degree of sphericity in pure water than vertically injected bubbles.

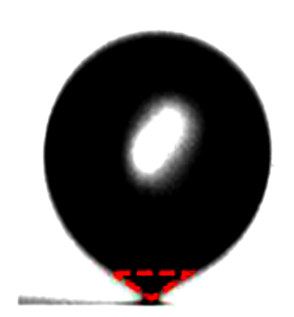

Conical zone

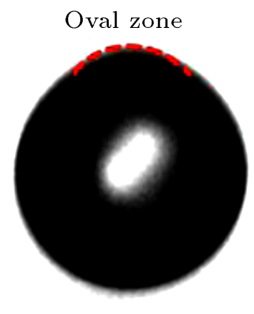

Figure 5. Bubble oscillation due to detachment from nozzle in pure water. 


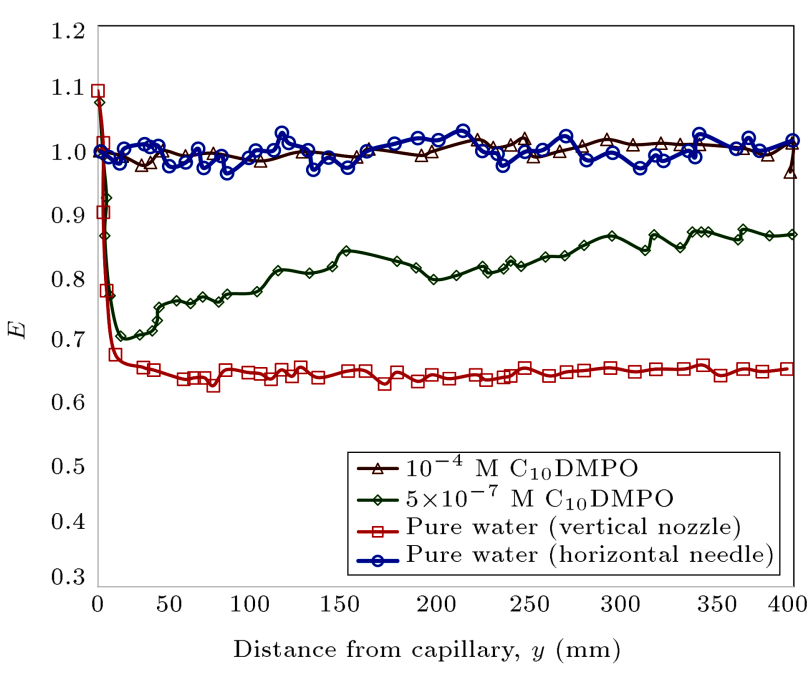

Figure 6. Aspect ratio of rising bubbles detached from vertical nozzle (in pure water and surfactant solutions) and horizontal needle (in pure water) as a function of the distance from the capillary.

\subsection{The effects of surfactants}

The aspect ratios of the bubbles detached from vertical nozzle in various surfactant solutions $\left(\mathrm{C}_{10} \mathrm{DMPO}\right.$ and SDS) are presented in Figure 7. By increasing the surfactant concentration, the aspect ratio at the detachment moment approaches 1, meaning that the detachment shape of the bubble tends toward a higher degree of sphericity. As shown by both Figure 7(a) and (b), the bubble aspect ratio descends from 1.1 to 0.65 in pure water; the same trend occurs for lower concentrations of the surfactant, yet with lower variations. However, for high surfactant concentrations $\left(10^{-4} \mathrm{M}\right.$ $\mathrm{C}_{10} \mathrm{DMPO}$ and $3 \times 10^{-3} \mathrm{M}$ SDS), the aspect ratio slightly fluctuates about unity. As can be observed for intermediate concentrations $\left(5 \times 10^{-7}-10^{-5} \mathrm{M}\right.$ $\mathrm{C}_{10} \mathrm{DMPO}$ and $5 \times 10^{-5}-3 \times 10^{-4} \mathrm{M}$ SDS $)$, there are minimums followed by the aspect ratio increase.

As mentioned earlier, the interfacial area of the surface-stretched bubble is higher than that of the

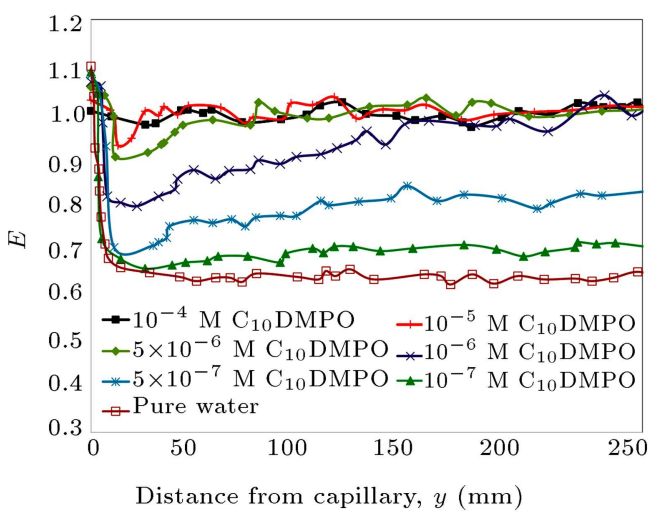

(a) spherical bubble of identical volume as well as bubble surface energy. Therefore, surface-stretched bubbles have greater initial instability and shape deformation than spherical ones do. The oscillation of bubbles induces some streamlines around them [38]. The formation of flow field due to the bubble oscillation could influence the local velocity of rising bubbles.

The LVPs of the bubbles detached from vertical nozzle in pure water and various surfactant solutions $\left(10^{-7} \mathrm{M}\right.$ to $10^{-4} \mathrm{M}$ of $\mathrm{C}_{10} \mathrm{DMPO}$ and $5 \times 10^{-5} \mathrm{M}$ to $3 \times 10^{-3} \mathrm{M}$ of $\mathrm{SDS}$ ) are shown in Figure 8 . By increasing the surfactant concentration, the terminal velocity decreases from $35 \mathrm{~cm} / \mathrm{s}$ to $15 \mathrm{~cm} / \mathrm{s}$. For intermediate concentrations $\left(5 \times 10^{-7} \mathrm{M}\right.$ to $10^{-5} \mathrm{M}$ for $\mathrm{C}_{10} \mathrm{DMPO}$ and $5 \times 10^{-5} \mathrm{M}$ to $3 \times 10^{-4} \mathrm{M}$ for $\left.\mathrm{SDS}\right)$, after the initial acceleration of the bubble, a maximum is observed followed by the velocity decrease up to terminal velocity.

This behavior is well described by the DAL theory. According to this theory, during the rise of bubbles through pure liquids, the gas-liquid interface is fully mobile and rising bubble velocity is higher than that of a rigid sphere of the same size [26,39]. In surfactant solutions, an adsorption layer is formed on the bubble surface, thus retarding the mobility of the interface and increasing the viscous drag [12]. The drag force exerted by the liquid medium leads to the development of nonuniform distribution of surface concentration on the bubble surface. Surface concentration of surfactants is at minimum at the leading pole of the rising bubble, whereas the rear pole is enriched from surfactant molecules [40]. Surface concentration gradient induces Marangoni stress that reduces the mobility of the bubble surface and lowers the bubble velocity $[9,41]$. When the bubble velocity approaches the terminal velocity, a stagnant cap is formed at the rear of the bubble, which is known as a diffusion adsorption layer. However, the effect of the initial shape of the detached bubble as a representative parameter of surface energy is disregarded in DAL theory.

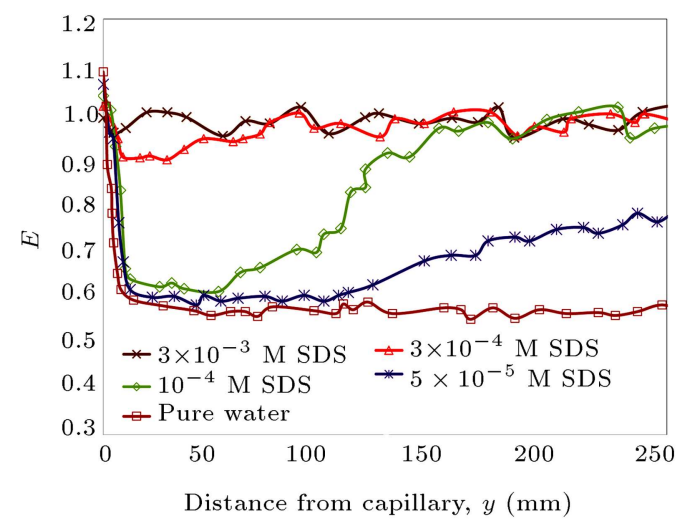

(b)

Figure 7. Aspect ratios of vertically injected bubbles in various solutions of (a) $\mathrm{C}_{10}$ DMPO, and (b) SDS. 


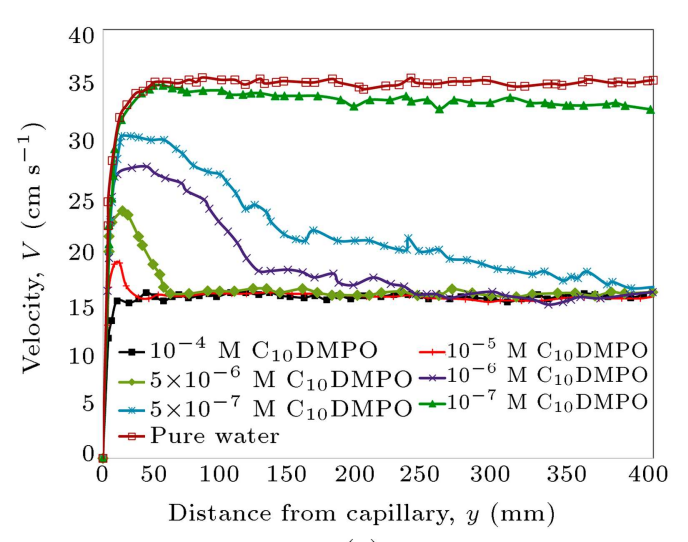

(a)

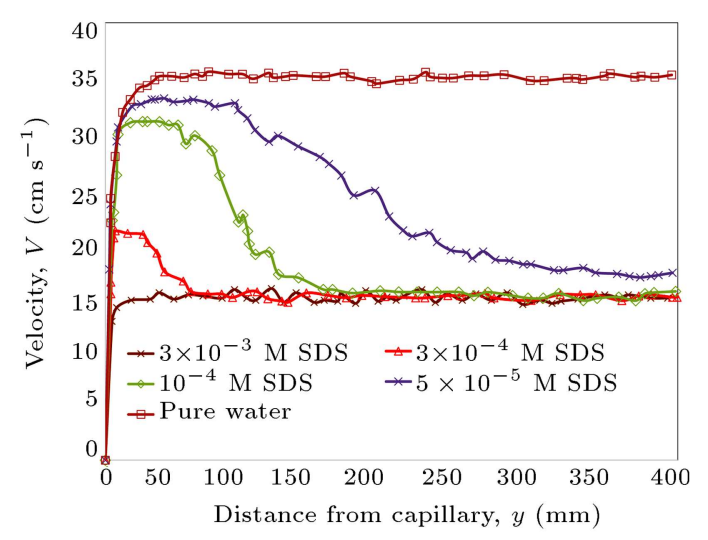

(b)

Figure 8. Local velocities of vertically injected bubbles in various solutions of (a) $\mathrm{C}_{10}$ DMPO, and (b) SDS.

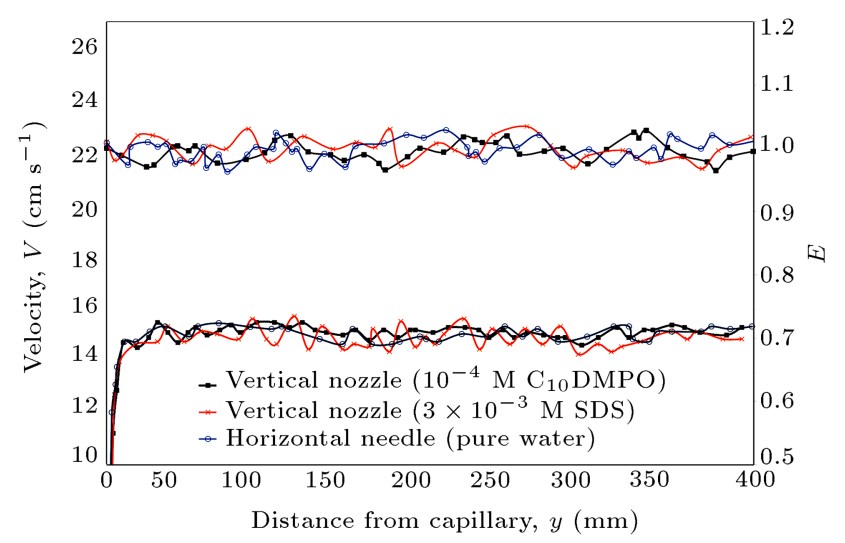

Figure 9. Velocity profile and aspect ratio of initially spherical bubble as a function of distance from the capillary.

For a better understanding, LVPs and aspect ratio of the rise of a horizontally detached bubble in pure water are compared to those of a vertically detached bubble in the concentrated surfactant solution $\left(3 \times 10^{-3}\right.$ M SDS and $\left.10^{-4} \mathrm{M} \mathrm{C}_{10} \mathrm{DMPO}\right)$, as shown in Figure 9.
In all cases of Figure 9, rising bubbles have identical behavior because of their same detachment shape of sphere. As was pointed out earlier, surfactants cause the reduction of bubble stretching curvature produced in vertical nozzle. Therefore, the initial shape oscillation is damped and, consequently, aspect ratio undergoes only slight changes in the rising path and terminal velocity is less than that of the surfacestretched bubble.

The presence of surfactant affects the velocity of the rising bubble by changing its initial shape. This is a supplementary point of view for velocity retardation of the rising bubble in the presence of a surfactant that differs from the creation of Marangoni stresses and the increase of hydrodynamic drag.

Aspect ratio versus Weber and Tadaki numbers are presented in Figure 10 (for various concentrations of $\mathrm{C}_{10} \mathrm{DMPO}$ ) as in a comparison with Eqs. (7) and (8). Both equations show good fitting with experimental data of $5 \times 10^{-7} \mathrm{M} \mathrm{C}_{10}$ DMPO. The results of Taylor \& Acrovis were disappointing for horizontally injected bubbles in pure water. Figure 11 shows the aspect

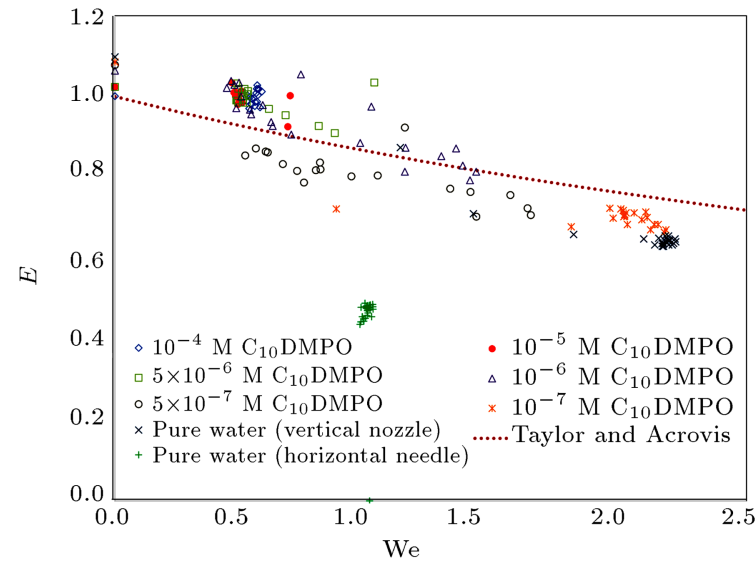

(a)

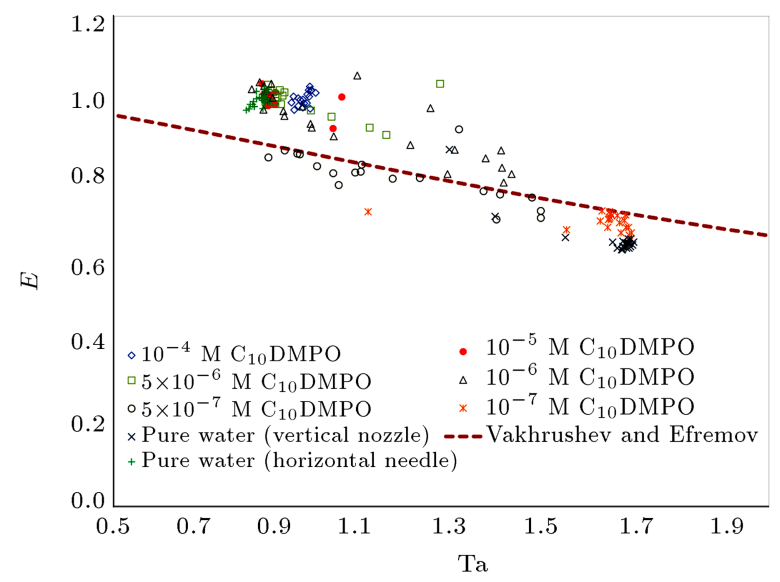

(b)

Figure 10. Aspect ratio of rising bubbles in terms of (a) We and (b) Ta for $\mathrm{C}_{10} \mathrm{DMPO}$ solutions. 


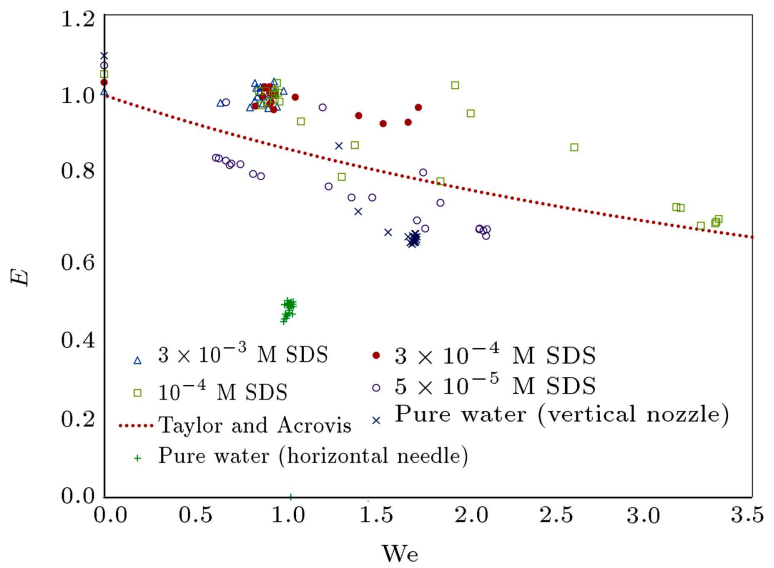

(a)

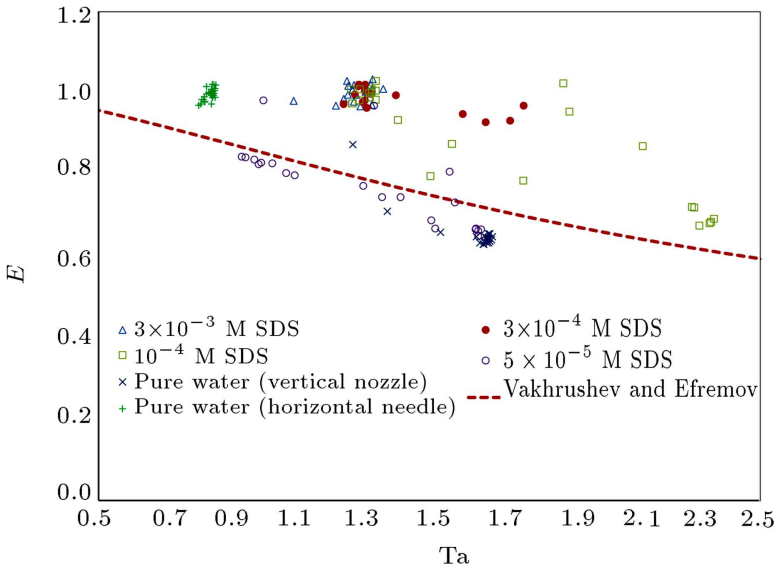

(b)

Figure 11. Aspect ratio of rising bubbles in terms of (a) We and (b) Ta for SDS solutions.

ratio in terms of Weber and Tadaki numbers for SDS solutions along with Eqs. (7) and (8). Good estimates of experimental data are provided for $5 \times 10^{-5}$ M SDS using Eqs. (7) and (8). Scattered data have been observed for $10^{-4} \mathrm{M}$ and higher concentrations of SDS. Generally, Vakhrushev \& Efremov model has been found to provide better estimates of the bubble aspect ratio.

\section{Conclusion}

The present study demonstrates a novel explanation for the rising bubble hydrodynamics in the presence of surfactants that emphasizes the initial shape of detached bubbles. Two types of capillaries were used for the bubble generation: one aligned horizontally and the other aligned vertically. Horizontally injected bubbles had an initial spherical shape, and their values of aspect ratio slightly fluctuated around unity. Thus, the bubble rises without significant deformation were compared to its initial spherical shape.

However, vertically injected bubbles had an initial surface-stretched shape in pure water, and their values of aspect ratios decreased abruptly from 1.1 to 0.65 .

The presence of surfactants decreased the surface tension of vertically injected bubbles and, accordingly, surface energy decreased. Increasing surfactant concentrations led to deformation of the initial curvature of vertically injected bubbles from an unstable surfacestretched shape (pure water) to a highly stable spherical shape $\left(3 \times 10^{-3} \mathrm{M}\right.$ SDS and $\left.10^{-4} \mathrm{M} \mathrm{C}_{10} \mathrm{DMPO}\right)$. According to the data of LVPs, there is a notable correspondence between the variation of local velocities and aspect ratios, which is relevant to the initial shape of the bubbles. This specific behavior reveals that surfactants could affect the rising bubble hydrodynamics by controlling the detachment shape of the bubble. Taylor \& Acrovis and Vakhrushev \& Efremov models were evaluated by using the experimental data for estimating the bubble aspect ratio in terms of Weber and Tadaki numbers, respectively. The results showed that Vakhrushev \& Efremov correlation provided better estimates of the bubble aspect ratio than Taylor \& Acrovis model did.

\section{Nomenclature}

a Long axis of the oval bubble

$b \quad$ Short axis of the oval bubble

$E \quad$ Aspect ratio

$E_{s} \quad$ Surface energy (j)

Re Reynolds number

We Weber number

Eo Eötvös number

Ta Tadaki number

$V \quad$ Local velocity $\left(\mathrm{cm} . \mathrm{s}^{-1}\right)$

$y \quad$ Distance from capillary $(\mathrm{mm})$

\section{Greek letters}

$\sigma \quad$ Surface tension (N.m ${ }^{-1}$ )

$\theta \quad$ Detachment angle (degree)

\section{References}

1. Ulaganathan, V., Krzan, M., Lotfi, M., et al. "Influence of $\beta$-lactoglobulin and its surfactant mixtures on velocity of the rising bubbles", Colloids Surfaces A Physicochem. Eng. Asp., 460, pp. 361-368 (2014). DOI:10.1016/j.colsurfa.2014.04.041

2. Lotfi, M., Karbaschi, M., Javadi, A., et al. "Dynamics of liquid interfaces under various types of external perturbations", Curr. Opin. Colloid Interface Sci., 19, pp. 309-319 (2014). DOI:10.1016/j.cocis.2014.04.006

3. Dukhin, S.S., Kovalchuk, V.I., Gochev, G.G., et al. "Dynamics of rear stagnant cap formation at the 
surface of spherical bubbles rising in surfactant solutions at large reynolds numbers under conditions of small Marangoni number and slow sorption kinetics", Adv. Colloid Interface Sci., 222, pp. 260-274 (2015). DOI:10.1016/j.cis.2014.10.002

4. Seddigh, E., Azizi, M., Sani, E.S., et al. "Investigation of poly(ether-b-amide)/nanosilica membranes for CO2/CH4 separation", Chinese J. Polym. Sci., 32, pp. 402-410 (2014). DOI:10.1007/s10118-014-1416-y

5. Paul, N., Schulz, J.M., and Kraume, M. "Fluid dynamics of droplets as a useful tool to determine coverage and adsorption kinetics of surfactants", Chem. Eng. Technol., 38, pp. 1979-1984 (2015). DOI:10.1002/ceat.201500137

6. Liu, Z., Herman, C., and Kim, J. "Heat transfer and bubble detachment in subcooled pool boiling from a downward-facing microheater array in a nonuniform electric field", Ann. N. Y. Acad. Sci., 1161, pp. 182191 (2009). DOI:10.1111/j.1749-6632.2008.04331 x.

7. Matavos-Aramyan S., Ghazi-MirSaeed M., SaeediEmadi, A., et al. "Influence of the process parameters on the foam fractionation treatment of olive mill wastewater", Sci. Iran., 23, pp. 2820-2827 (2016). DOI:10.24200/sci.2016.3992

8. Papadopoulou, V., Tang, M.-X., Balestra, C., et al. "Circulatory bubble dynamics: From physical to biological aspects", Adv. Colloid Interface Sci., 206, pp. 239-249 (2014). DOI:10.1016/j.cis.2014.01.017

9. Zawala, J., Kosior, D., and Malysa, K. "Formation and influence of the dynamic adsorption layer on kinetics of the rising bubble collisions with solution/gas and solution/solid interfaces", Adv. Colloid Interface Sci., 222, pp. 765-778 (2015). DOI:10.1016/j.cis.2014.07.013

10. Shahid, M., Fan, C., and Pashley, R.M. "Insight into the bubble column evaporator and its applications", Int. Rev. Phys. Chem., 35, pp. 143-185 (2016). DOI:10.1080/0144235X.2016.1147144

11. Saffman, P.G. and Sears, W.R. "On the rise of small air bubbles in water", J. Fluid Mech., 1, p. 249 (1956). DOI:10.1017/S0022112056000159

12. Dukhin, S.S., Miller, R., and Loglio, G., Physicochemical Hydrodynamics of Rising Bubble, Elsevier, pp. 367-432 (1998). DOI:10.1016/S13837303(98)80025-2

13. Shoghl, S.N., Bahrami, M., and Moraveji, M.K. "Experimental investigation and CFD modeling of the dynamics of bubbles in nanofluid pool boiling", Int. Commun. Heat Mass Transf., 58, pp. 12-24 (2014). DOI:10.1016/j.icheatmasstransfer.2014.07.027

14. Chakraborty, I., Biswas, G., Polepalle, S., et al. "Bubble formation and dynamics in a quiescent highdensity liquid", AIChE J., 61, pp. 3996-4012 (2015). DOI:10.1002/aic.14896

15. Azizi, M., Ramazani, A., Etemadi, H., et al. "Simulation of viscoelastic fluid flows in expansion geometry using finite volume approach", Chinese J. Polym. Sci., 31, pp. 1599-1612 (2013). DOI:10.1007/s10118-013$1336-2$
16. Carvajal, D., Carlesi, C., Meléndez-Vejar, V., et al. "Numerical simulation of single-bubble dynamics in high-viscosity ionic liquids using the levelset method", Chem. Eng. Technol., 38, pp. 473-481 (2015). DOI:10.1002/ceat.201400449

17. Premlata, A.R., Tripathi, M.K., and Sahu, K.C. "Dynamics of rising bubble inside a viscositystratified medium", Phys. Fluids., 27072105 (2015). DOI:10.1063/1.4927521

18. Ohta, M., Tsuji, M., Yoshida, Y., et al. "The transient dynamics of a small bubble rising in a low Morton number regime", Chem. Eng. Technol., 31, pp. 13501357 (2008). DOI:10.1002/ceat.200700507

19. Tomiyama, A., Celata, G.P., Hosokawa, S., et al. "Terminal velocity of single bubbles in surface tension force dominant regime", Int. J. Multiph. Flow., 28, pp. 1497-1519 (2002). DOI:10.1016/S0301-9322(02)000320

20. Krzan, M. and Malysa, K. "Profiles of local velocities of bubbles in n-butanol, n-hexanol and n-nonanol solutions", Colloids Surfaces A Physicochem. Eng. Asp., 207, pp. 279-291 (2002). DOI:10.1016/S0927$7757(02) 00163-2$

21. Ziegenhein, T. and Lucas, D. "Observations on bubble shapes in bubble columns under different flow conditions", Exp. Therm. Fluid Sci., 85, pp. 248-256 (2017). DOI:10.1016/j.expthermflusci.2017.03.009

22. Dukhin, S.S., Lotfi, M., Kovalchuk, V.I., et al. "Dynamics of rear stagnant cap formation at the surface of rising bubbles in surfactant solutions at large Reynolds and Marangoni numbers and for slow sorption kinetics", Colloids Surfaces A Physicochem. Eng. Asp., 492, pp. 127-137 (2016). DOI:10.1016/j.colsurfa.2015.12.028

23. Malysa, K., Krasowska, M., and Krzan, M. "Influence of surface active substances on bubble motion and collision with various interfaces", $A d v$. Colloid Interface Sci., 114, pp. 205-225 (2005). DOI:10.1016/j.cis.2004.08.004

24. Bastani, D., Fayzi, P., Lotfi, M., et al. "CFD simulation of bubble in flow field: Investigation of dynamic interfacial behaviour in presence of surfactant molecules", Colloids Interface Sci. Commun., 27, pp. 1-10 (2018). DOI:10.1016/j.colcom.2018.09.001

25. Lotfi, M., Bastani, D., Ulaganathan, V., et al. "Bubble in flow field: A new experimental protocol for investigating dynamic adsorption layers by using capillary pressure tensiometry", Colloids Surfaces A Physicochem. Eng. Asp., 460, pp. 369-376 (2014). DOI:10.1016/j.colsurfa.2013.11.011

26. Clift, R., Grace, J.R., and Weber, M.E., Bubbles, Drops, and Particles, Academic Press, 2005 (Accessed: April 25, 2017). 
27. Ellingsen, K. and Risso, F. "On the rise of an ellipsoidal bubble in water: Oscillatory paths and liquidinduced velocity", J. Fluid Mech., 440, pp. 235-268 (2001). DOI:10.1017/S0022112001004761

28. Bhaga, D. and Weber, M.E. "Bubbles in viscous liquids: Shapes, wakes and velocities", J. Fluid Mech., 105, pp. 61-85 (1981). DOI:10.1017/S002211208100311X

29. Wu, M. and Gharib, M. "Experimental studies on the shape and path of small air bubbles rising in clean water", Phys. Fluids., 14, L49 (2002). DOI:10.1063/1.1485767

30. Celata, G.P., D'Annibale, F., Di Marco, P., et al. "Measurements of rising velocity of a small bubble in a stagnant fluid in one and two-component systems", Exp. Therm. Fluid Sci., 31, pp. 609-623 (2007). DOI:10.1016/j.expthermflusci.2006.06.006

31. Tomiyama, A., Kataoka, I., Zun, I., et al. "Drag coefficients of single bubbles under normal and micro gravity conditions", JSME Int. J. Ser. B., 41, pp. 472479 (1998). DOI:10.1299/jsmeb.41.472

32. Vafaei, S., Angeli, P., and Wen, D. "Bubble growth rate from stainless steel substrate and needle nozzles", Colloids Surfaces A Physicochem. Eng. Asp., 384, pp. 240-247 (2011). DOI:10.1016/j.colsurfa.2011.03.066

33. Hashmi, A., Yu, G., Reilly-Collette, M., et al. "Oscillating bubbles: A versatile tool for lab on a chip applications", Lab Chip., 12, p. 4216 (2012). DOI:10.1039/c2lc40424a

34. Tesar̆, V. "Shape oscillation of microbubbles", Chem. Eng. J., 235, pp. 368-378 (2014). DOI:10.1016/j.cej.2013.09.027

35. Krzan, M., Zawala, J., and Malysa, K. "Development of steady state adsorption distribution over interface of a bubble rising in solutions of n-alkanols (C5, C8) and n-alkyl trimethyl ammonium bromides (C8, C12, C16)", Colloids Surfaces A Physicochem. Eng. Asp., 298, pp. 42-51 (2007). DOI:10.1016/j.colsurfa.2006.12.056

36. Taylor, T.D. and Acrivos, A. "On the deformation and drag of a falling viscous drop at low Reynolds number", J. Fluid Mech., 18, pp. 466-476 (1964). DOI:10.1017/S0022112064000349

37. Vakhrushev, I.A. and Efremov, G.I. "Interpolation formula for computing the velocities of single gas bubbles in liquids", Chem. Technol. Fuels Oils., 6, pp. 376-379 (1970). DOI:10.1007/BF01171684

38. Marmottant, P. and Hilgenfeldt, S. "Controlled vesicle deformation and lysis by single oscillating bubbles", Nature., 423, pp. 153-156 (2003).

DOI:10.1038/nature01613

39. Hadamard, J.S. "Hydrodynamics - On a question relating to the viscous liquid" [Hydrodynamique-sur une question relative aux liquides visqueux], Comptes Rendus., 154, p. 109 (1912).
40. Dukhin, S.S., Kretzschmar, G., and Miller, R. Thermodynamics and Macro-kinetics of Adsorption, Elsevier (1995). DOI:10.1016/S1383-7303(06)80010-4

41. Saidi, M.H., Taeibi-Rahni, M., Asadi, B., et al. "Computational simulation of marangoni convection under microgravity condition", Sci. Iran., Trans. BMechanical Eng., 16, pp. 513-524 (2009).

\section{Biographies}

Pouyan Fayzi received his BSc and MSc degrees in 2010 and 2012 from University of Sistan and Baluchestan, Zahedan, Iran, and Shiraz University, School of Chemical, Petroleum \& Gas Engineering, Shiraz, Iran, all in Chemical Engineering, respectively. Mr. Fayzi is currently a PhD Candidate in Chemical Engineering at Chemical and Petroleum Department of Sharif University of Technology, Tehran, Iran. His research interests are interfacial phenomena and multiphase flow in the presence of surface active materials.

Dariush Bastani received his BSc degree from Sharif University of Technology in Chemical Engineering. He also received his $\mathrm{MSc}$ degrees and $\mathrm{PhD}$ degrees from University of Manchester Institute of Science and Technology, Manchester, United Kingdom, all in Chemical Engineering. Dr. Bastani is currently a Professor of Chemical Engineering at Chemical and Petroleum Department of Sharif University of Technology, Tehran, Iran. His research interests are separation processes, liquid-liquid extraction, and interfacial phenomena.

Marzieh Lotfi received her BSc and MSc degrees in 2008 and 2010 from Amir Kabir University of Technology, Department of Chemical Engineering, Tehran, Iran, all in Chemical Engineering, and received her PhD in 2015 from Sharif University of Technology Chemical and Petroleum Department, Tehran, Iran, in Chemical Engineering. Dr. Lotfi is currently an Assistant Professor of Chemical Engineering in Science and Research Branch, Islamic Azad University, Department of Chemical Engineering, Tehran, Iran. Her research interests are interfacial phenomena and multi-phase flow in the presence of surface active materials.

Mohammad Ghamangiz Khararoodi received his BSc and MSc degrees in 2014 and 2016 from University of Tehran, Caspian Faculty of Engineering, Gilan, Iran, and Sharif University of Technology, Chemical and Petroleum Department, Tehran, Iran, all in Chemical Engineering, respectively. His research interest is interfacial phenomena. 